\title{
Treatment of large avascular retinal pigment epithelium detachments in age-related macular degeneration with aflibercept, photodynamic therapy, and triamcinolone acetonide
}

This article was published in the following Dove Medical Press journal:

Clinical Ophthalmology

\author{
Arnt-Ole Tvenning ${ }^{1,2}$ \\ Christian Hedels ${ }^{3}$ \\ Jørgen Krohn ${ }^{4,5}$ \\ Dordi Austeng ${ }^{1,2}$ \\ 'Department of Neuromedicine \\ and Movement Science, Faculty of \\ Medicine and Health Sciences, NTNU, \\ Norwegian University of Science and \\ Technology, 749I Trondheim, Norway; \\ ${ }^{2}$ Department of Ophthalmology, \\ St. Olavs Hospital, Trondheim \\ University Hospital, 7006 Trondheim, \\ Norway; ${ }^{3}$ Hedels Eye Clinic, 6517 \\ Kristiansund, Norway; ${ }^{4}$ Department \\ of Clinical Medicine, Section of \\ Ophthalmology, University of Bergen, \\ 502 I Bergen, Norway; ${ }^{5}$ Department of \\ Ophthalmology, Haukeland University \\ Hospital, 5053 Bergen, Norway
}

Purpose: To evaluate the use of aflibercept, triamcinolone acetonide, and photodynamic therapy (PDT) in the treatment of avascular pigment epithelium detachments (aPEDs).

Patients and methods: Patients with treatment-naïve aPEDs $\geq 1,500 \mu \mathrm{m}$ in diameter were randomized to treatment or observation. Treatment consisted of 6 monthly intravitreal injections of aflibercept. If the aPED persisted, the patients were treated with half-fluence PDT in combination with intravitreal triamcinolone acetonide and aflibercept. The primary outcome was change of best-corrected visual acuity (BCVA) after 24 months of follow-up. Secondary outcomes were changes in pigment epithelium volume, height and diameter, central retinal thickness, and number of patients developing choroidal neovascularization or geographic atrophy (GA). Results: Treatment and inclusion of patients were stopped after an interim analysis of 6-month data because $75 \%$ of the aPEDs were in different stages of GA. Nine patients with aPED were included in the study, of these one patient was excluded because of bilateral central serous chorioretinopathy. The remaining eight had drusenoid aPEDs. After 24 months of follow-up, estimated means of BCVA decreased by 4.2 and 20.8 letters in the treatment and observation group, respectively. This decrease over time was not significantly different between groups ( $P=0.140,95 \% \mathrm{CI}-5.3,38.6)$. Estimated means of PED volume, height, diameter, and central retinal thickness were not significantly different between groups. Choroidal neovascularization and retinal pigment epithelium tear developed in one patient in the treatment group. One patient in the treatment group and two patients in the observation group progressed to complete retinal pigment epithelium and outer retinal atrophy. A decrease in PED volume was associated with the development of complete retinal pigment epithelium and outer retinal atrophy $(P=0.029)$.

Conclusion: This small trial indicates that multitargeted, primarily antiangiogenic therapy does not favorably alter the natural course of drusenoid aPEDs.

Keywords: drusenoid, geographic atrophy, anti-VEGF, PDT

\section{Plain language summary}

How to manage large accumulations of lipids and fluid in the part of your eye responsible for good vision, without associated leaking blood vessels, is not fully understood. Their presence is associated with a risk of visual impairment. Available treatments (anti-vascular endothelial growth factor and photodynamic therapy) have in small studies and case reports shown some beneficial effects.

We investigated if treatment could be beneficial by designing a study with patients randomly assigned to treatment or observation. Treatment consisted of injecting the eye with anti-vascular
Correspondence: Arnt-Ole Tvenning ment of Neuromedicine and Movement Science, Faculty of Medicine and Health Sciences, NTNU, Norwegian University of Science and Technology, P.O. Box 8905, 749I Trondheim, Norway Tel +47 72575012

Fax +4772574833

Email arnt.o.tvenning@ntnu.no 
endothelial growth factor monthly for 6 months, and then adding photodynamic therapy and injections of corticosteroids if the accumulation of lipids and fluid did not resolve. Our study was stopped early when 6 months data were available because $75 \%$ of the patients progressed to a more advanced form of the disease with no currently available treatment.

This small study indicates that treatment with a combination of anti-vascular endothelial growth factor, corticosteroid, and photodynamic therapy does not favorably alter the natural course of large accumulations of lipids and fluid in age-related macular degeneration without associated leaking blood vessels.

\section{Introduction}

Age-related macular degeneration (AMD) is the most common cause of permanent vision loss in the industrialized world. ${ }^{1-3}$ With the introduction of anti-vascular endothelial growth factor (anti-VEGF) agents, treatment for neovascular AMD (nAMD) has undergone significant advances. There are still dilemmas to be solved, and research on biomarkers relevant for visual function, disease activity, and prognosis is desirable. ${ }^{4}$ One such biomarker, pigment epithelium detachment (PED), is still a challenge, both in avascular PED (aPED) and when neovascularization is present (nPED). The pathophysiologic mechanisms underlying the development of PEDs are not fully understood, and probably represent a continuum of degenerative changes and formation of choroidal neovascularization $(\mathrm{CNV}) .{ }^{5}$ Serous aPEDs are thought to arise from age-related formation of a hydrophobic barrier in Bruch's membrane preventing the free diffusion of fluid from retinal pigment epithelium (RPE) to choriocapillaris. ${ }^{6}$ Drusenoid aPED formation is a result of the confluence of large drusen, and probably with increasing size the hydrophobic composition of the drusenoid deposits also creates a barrier in Bruch's membrane with accumulation of fluid. ${ }^{7,8}$

In some retrospective case series, nPEDs respond well to treatment with anti-VEGF. ${ }^{9-11}$ In other studies, the results are not so promising, ${ }^{12}$ and nPEDs might be more resistant to treatment. ${ }^{4}$ Nonresponders to anti-VEGF treatment have the option of photodynamic therapy (PDT), which has been shown to stabilize disease progression, ${ }^{13}$ but the treatment may lead to chorioretinal atrophy and decline in visual acuity. ${ }^{14}$

The natural course of aPED is unfavorable. In the AgeRelated Eye Disease Study, it was found that $42 \%$ of 282 eyes with drusenoid aPEDs progressed to advanced AMD, with either central geographic atrophy (GA) (19\%) or nAMD $(23 \%) .{ }^{15}$ In one study with long follow-up of 12 eyes with drusenoid aPED, all patients developed advanced AMD, with a 10 -year occurrence rate of $75 \%$ for central GA and $25 \%$ for
nAMD. ${ }^{8}$ Patients presenting with aPEDs remain a diagnostic and therapeutic challenge, and treatment is controversial. ${ }^{12}$ One prospective case series treated 12 drusenoid and serous aPEDs with anti-VEGF, which led to stabilization of bestcorrected visual acuity (BCVA) after 1 year, but with heterogeneous anatomical outcomes. ${ }^{16}$ In another prospective case series, six patients with drusenoid aPEDs were treated with anti-VEGF agents that led to stabilization of BCVA and improvement in 33\% of patients. ${ }^{17}$ Two case reports have reported regression of drusen and drusenoid PEDs following anti-VEGF treatment for associated CNV. ${ }^{18,19}$ Other case reports have shown regression of drusenoid aPED and improvement of BCVA after PDT. ${ }^{20,21}$

In the present study, we planned to evaluate the treatment of serous and drusenoid aPEDs. Our original hypothesis was that some aPEDs may be caused by subtle CNV, undetectable with today's technology, and that early treatment could be beneficial. To our knowledge, this is the first randomized controlled trial evaluating treatment of aPEDs with aflibercept, PDT, and triamcinolone acetonide.

\section{Patients and methods}

The study was conducted at the Department of Ophthalmology, Trondheim University Hospital, and the inclusion of patients started in April 2014. Patients with AMD and large PEDs, that on clinical evaluation seemed to be avascular, were referred from other eye departments and ophthalmologists in the Central Norway Health Region. The study is registered at ClinicalTrials.gov: NCT01746875, and was approved by the Regional Committee for Medical and Health Research Ethics Central Norway (2012/1743). All study participants provided a written informed consent, and the study was conducted in accordance with the Declaration of Helsinki.

Inclusion criteria were patients older than 50 years with treatment-naïve AMD, fluorescein angiographic (FA)- and indocyanine green angiographic (ICG)-verified subfoveal aPED $\geq 1,500 \mu \mathrm{m}$ in diameter, and BCVA $\leq 20 / 32$ and $\geq 20 / 400$. Exclusion criteria were subfoveal fibrosis, central GA, and concurrent eye diseases that could affect BCVA.

The diagnosis of aPED was made by FA, ICG, and spectral-domain optical coherence tomography (OCT). PEDs with no leakage on FA and an absence of plaques and hot spots on ICG were defined as aPEDs. The aPEDs were defined as drusenoid if they appeared yellow on clinical fundus examination with predominantly hyper-reflective contents on OCT, staining/pooling on FA, and hypofluorescence on ICG. 
The aPEDs were defined as serous if they appeared as domeshaped with predominantly hyporeflective contents on OCT, pooling on FA, and hypofluorescence on ICG. Central GA was defined as an area with window defects on FA and ICG, sharply delineated margins on fundus photography with visible choroidal vasculature, loss of a hyper-reflective RPE line, and increased choroidal reflectivity on OCT, hypofluorescence on fundus autofluoresence (FAF), and subfoveal localization.

Fundus and angiographic images (Zeiss FF 450 plus, Carl Zeiss Meditec AG, Jena, Germany) were reviewed and graded by two retina specialists (AT and DA). To ensure a correct diagnosis, all the images were also anonymized and sent for evaluation to an external retina specialist $(\mathrm{CH})$ prior to randomization. Randomization was performed by a web-based randomization system developed and administered by Unit of Applied Clinical Research, The Faculty of Medicine, Norwegian University of Science and Technology, Trondheim, Norway. The patients were stratified on the presence of drusenoid aPED.

BCVA was measured using Early Treatment Diabetic Retinopathy Study (ETDRS) chart in an illuminated Cabinet (ESV3000; Good-Lite, Elgin, IL, USA) at 4 m, with automatic adjustment of $85 \mathrm{~cd} / \mathrm{m}^{2}$. Room lighting was dimmed so that 90-110 lux could be measured in front of the cabinet. The examiners were blinded for patient group. Refraction was performed by a physician or optician prior to BCVA measurement at baseline, 1 year, and 2 years. Maximum PED height and diameter were measured by OCT (Cirrus HD-OCT; Carl Zeiss Meditec AG) on five different slides on macular cube scans starting in the central fovea $(512 \times 128)$, and the mean of the three highest values was used. PED volume was calculated after manual segmentation and subtracting of total retinal and $\mathrm{PED}$ volume, measured from the internal limiting membrane (ILM) to the fit-RPE line, with retinal volume, measured from ILM to RPE line (Figure 1). Central retinal thickness (CRT) was obtained automatically after manual segmentation of RPE and ILM.
Patients randomized to the treatment group received three monthly intravitreal injections of aflibercept $2 \mathrm{mg} / 0.05 \mathrm{~mL}$ (Eylea; Regeneron Inc., Tarrytown, NY, USA), with 3 additional monthly injections if the PED persisted at 3 months. If the PED did not completely resolve as measured by OCT, the patients were treated with half-fluence PDT in combination with aflibercept $2 \mathrm{mg} / 0.05 \mathrm{~mL}$ and triamcinolone acetonide $4 \mathrm{mg} / 0.1 \mathrm{~mL}$ (Triesence; Alcon lab Inc., Forthworth, TX, USA) at 6 and 9 months (triple-therapy). The half-fluence PDT treatment protocol consisted of intravenous administration of $15 \mathrm{mg}$ Verteporfin (Visudyne; Bausch and Lomb Inc., Bridgewater, NJ, USA) in a $30 \mathrm{~mL}$ solution over 10 minutes, and after a waiting period of 5 minutes, infrared light (689 $\mathrm{nm}$ ) with a spot size of 3,000-4,000 $\mu \mathrm{m}$ was applied for 83 seconds with $25 \mathrm{~J} / \mathrm{cm}^{2}$ and $300 \mathrm{~mW} / \mathrm{cm}^{2}$ (Visulas $690 \mathrm{~s}$, Carl Zeiss Meditec AG). Intravitreal injections of aflibercept and triamcinolone acetonide were given on separate days 0-2 weeks after the PDT treatment. Additional follow-up examinations were performed at 6-month intervals in both groups and included BCVA measurements, OCT, fundus photography, FA, ICG, and FAF.

The primary outcome measure was changes in BCVA from baseline to 24 months. Secondary outcome measures were changes in PED height, diameter and volume, CRT, and number of patients with progression of GA or development of CNV. Progression of GA was graded as described by Sadda et $\mathrm{al}^{22}$ : complete RPE and outer retinal atrophy (cRORA); 1) region of hypertransmission of at least $250 \mu \mathrm{m}$ in diameter in any lateral dimension, 2) zone of attenuation or disruption of the RPE of at least $250 \mu \mathrm{m}$ in diameter, 3 ) evidence of overlying photoreceptor degeneration. Features of photoreceptor degeneration include all of the following: loss of interdigitation zone, ellipsoid zone, and external limiting membrane, and thinning of the outer nuclear layer, which also can be identified by a descending outer plexiform layer. In case of incomplete RPE and outer retinal atrophy (iRORA), the criteria for cRORA are not met, but hypertransmission and
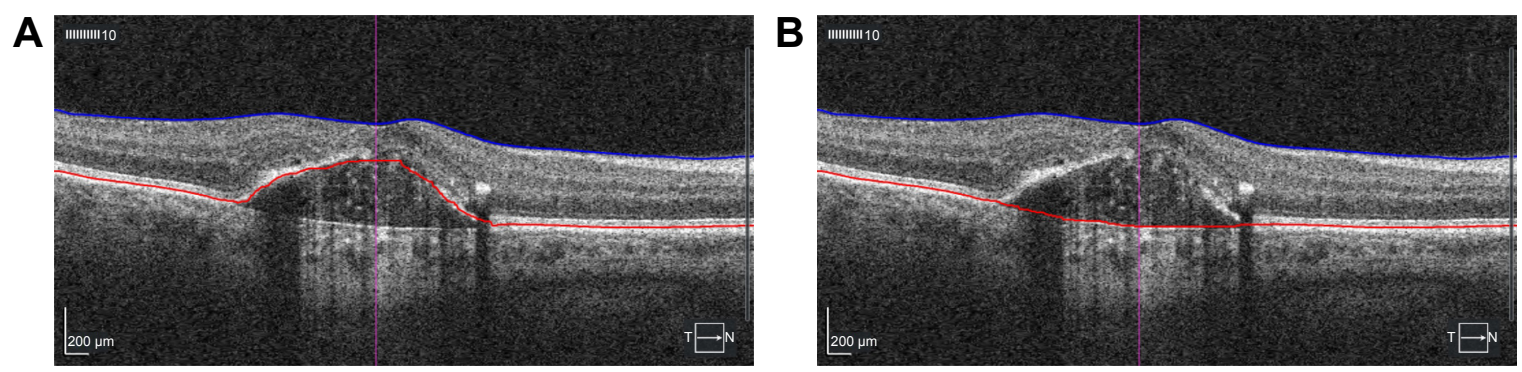

Figure I Manual segmentation and volume measurement of retinal pigment epithelium detachment from macular cube $5 \mathrm{I} 2 \times 128$ scans.

Notes: (A) Segmentation of internal limiting membrane and retinal pigment epithelium. (B) Segmentation of internal limiting membrane to fit line of retinal pigment epithelium. 
a discontinuous RPE band with evidence of photoreceptor degeneration are found. Exclusion criteria were presence of scrolled RPE or other signs of an RPE tear.

A sample size of 60 patients was decided to be sufficient to detect a clinically meaningful difference. Marginal models with restricted maximum likelihood estimation and unstructured or exponential covariance structure were used. All marginal models had age, baseline value, group, time, and the interaction between group and time as predictors. The $P$-values and $95 \%$ CIs of the comparisons of estimated means were Sidak adjusted because of multiple comparisons. Model fit was evaluated with Akaike's information criterion and likelihood ratio tests. The residuals were checked for normality compared to the normal distribution assessed with histograms, Q-Q plots, and Shapiro-Wilks test. Two-tailed $P$-values $<0.05$ were considered statistically significant. SPSS statistics 25 (IBM) and Stata 15.1 (StataCorp LLC) were used for statistical analysis.

\section{Results}

A total of 14 patients with AMD and aPED $\geq 1,500 \mu \mathrm{m}$ in diameter were referred from April 2014 to February 2015. Of these, five patients did not meet the inclusion criteria. Two patients with serous PED, one with fibrovascular PED and one with drusenoid PED, had CNV. One patient had adult-onset foveomacular vitelliform dystrophy. Thus, nine patients with aPEDs were included in the study, with five randomized to the treatment group. One patient from the treatment group was excluded after 3 months of follow-up because of disappearance of bilateral serous aPEDs after tapering of systemic corticosteroid therapy on suspicion of central serous chorioretinopathy. Baseline characteristics of the eight drusenoid aPEDs are summarized in Table 1.

An interim analysis was performed after 6 months of follow-up. Because examination of the OCT images showed that the patients in both the treatment and observation groups were in different stages of GA formation and progression (Figure 2), it was decided to stop further inclusion and treatment of patients.

The four participants in the treatment group received 6 monthly injections of aflibercept, but further treatment differed. Two patients (Figure 2, ID: 2 and 3) received both treatments with triple-therapy, one patient had only one treatment (Figure 2, ID: 7) and one patient did not receive tripletherapy (Figure 2, ID: 5). All patients were followed for 12 months (mean 12.4, range 12-13), and three patients in each group had a follow-up of 24 months (mean 24.2, range 24-25).

\section{Best-corrected visual acuity}

After 24 months of follow-up, estimated means of BCVA decreased by 4.2 ETDRS letters and 20.8 ETDRS letters in the treatment and observation groups, respectively (Figure 3A). The loss of ETDRS letters from 6 to 24 months did not differ significantly between groups ( $P=0.140,95 \% \mathrm{CI}-5.3,38.6)$. After 6, 12, and 24 months of follow-up, the difference of BCVA was 0.8 ETDRS letters $(P=1.0,95 \% \mathrm{CI}-21.3$, 22.8), 0.3 ETDRS letters ( $P=1.0,95 \% \mathrm{CI}-21.8,22.3)$, and 17.4 ETDRS letters $(P=0.478,95 \% \mathrm{CI}-7.7,42.4)$ between the treatment and observation groups, respectively. Followup duration and age of patients were significant predictors of decreasing BCVA ( $P=0.048$ and $P=0.009$, respectively). BCVA at baseline was not a significant predictor $(P=0.593)$.

\section{PED volume}

After 24 months of follow-up, estimated means of PED volume increased by $0.13 \mathrm{~mm}^{3}$ in the treatment group and decreased by $0.13 \mathrm{~mm}^{3}$ in the observation group (Figure 3B). The change of PED volume from 6 to 24 months did not differ significantly between the groups ( $P=0.433,95 \%$ CI $-0.40,0.91)$. After 6,12 , and 24 months of follow-up, differences in PED volume were $0.22 \mathrm{~mm}^{3}(P=1.0,95 \%$

Table I Baseline characteristics of drusenoid aPEDs

\begin{tabular}{|c|c|c|c|c|c|c|c|c|c|c|}
\hline & \multicolumn{5}{|c|}{ Treatment $(n=4)$} & \multicolumn{5}{|c|}{ Observation $(n=4)$} \\
\hline & Mean & SD & Median & Min & Max & Mean & SD & Median & Min & Max \\
\hline Age, years & 76.8 & 7.4 & 76 & 69 & 86 & 73.8 & 7.8 & 74 & 64 & 83 \\
\hline BCVA, ETDRS letters & 65.0 & 7.0 & 65.0 & 58 & 72 & 66.8 & 6.5 & 70.0 & 57 & 70 \\
\hline $\begin{array}{l}\text { PED } \\
\text { Height, } \mu \mathrm{m} \\
\text { Diameter, } \mu \mathrm{m} \\
\text { Volume, } \mathrm{mm}^{3}\end{array}$ & $\begin{array}{l}279 \\
2,657 \\
0.98\end{array}$ & $\begin{array}{l}106 \\
1,124 \\
0.83\end{array}$ & $\begin{array}{l}284 \\
2,243 \\
0.65\end{array}$ & $\begin{array}{l}160 \\
1,848 \\
0.40\end{array}$ & $\begin{array}{l}387 \\
4,293 \\
2.20\end{array}$ & $\begin{array}{l}345 \\
2,275 \\
0.90\end{array}$ & $\begin{array}{l}53 \\
258 \\
0.50\end{array}$ & $\begin{array}{l}338 \\
2,276 \\
0.75\end{array}$ & $\begin{array}{l}298 \\
2,012 \\
0.50\end{array}$ & $\begin{array}{l}406 \\
2,535 \\
1.60\end{array}$ \\
\hline $\mathrm{CRT}, \mu \mathrm{m}$ & 218 & 3.7 & 219 & 213 & 222 & 199 & 12.7 & 204 & 180 & 208 \\
\hline
\end{tabular}

Abbreviations: aPED, avascular pigment epithelium detachment; BCVA, best-corrected visual acuity; CRT, central retinal thickness; ETDRS, Early Treatment Diabetic Retinopathy Study; n, number of patients; PED, pigment epithelium detachment. 

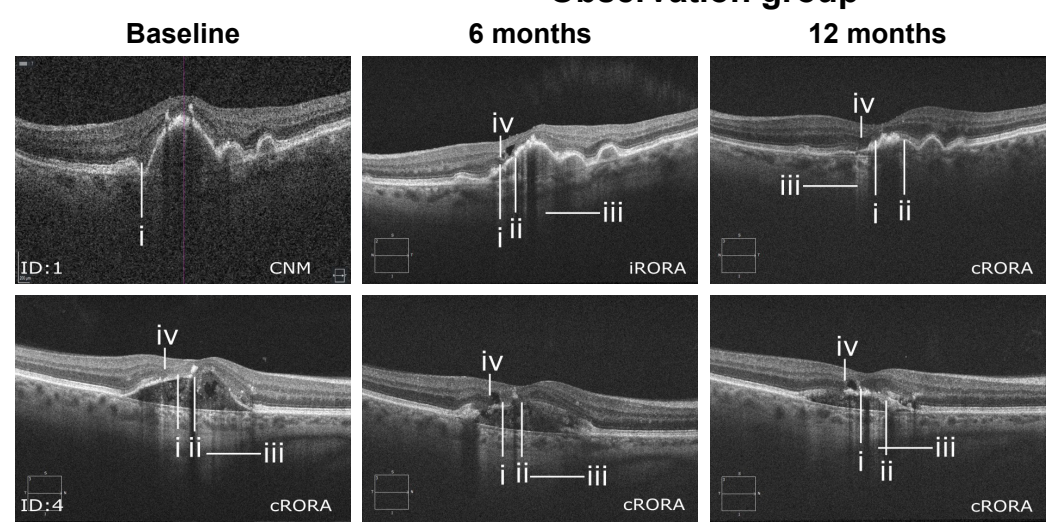

24 months
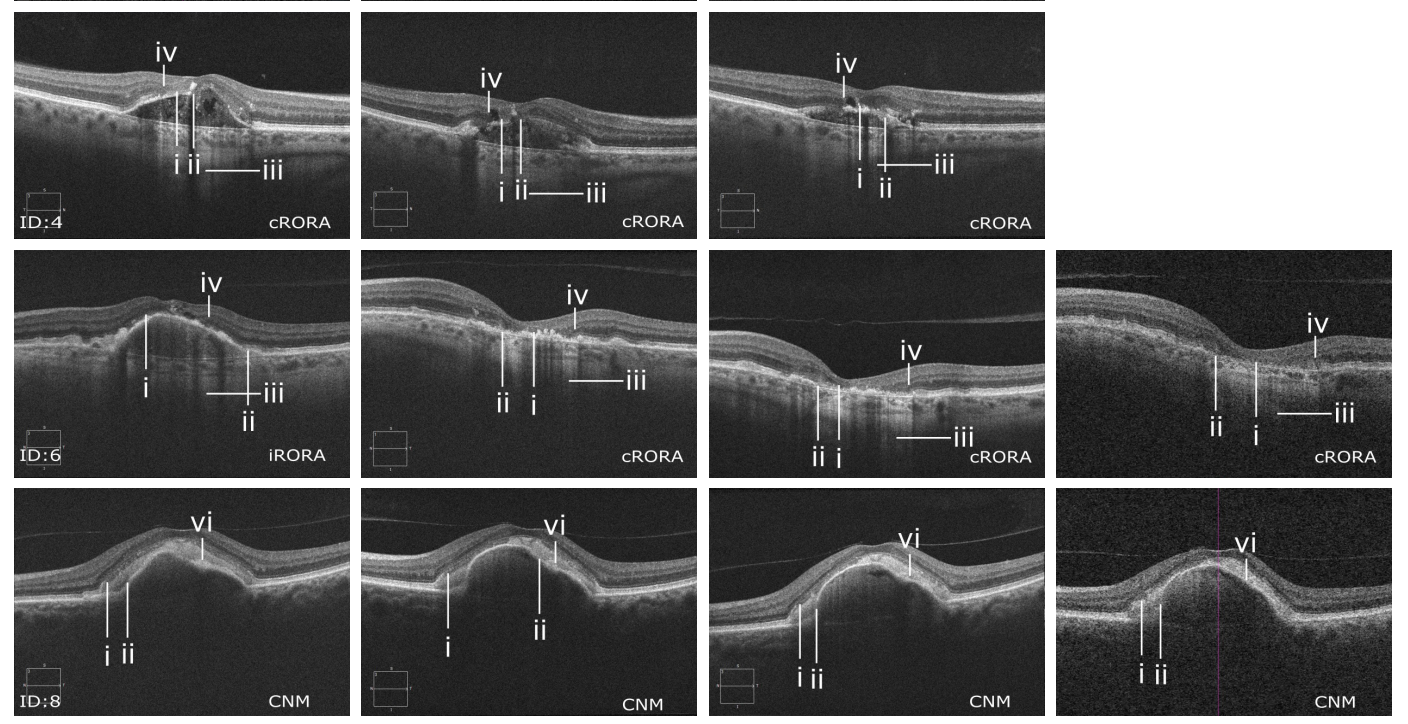

Treatment group
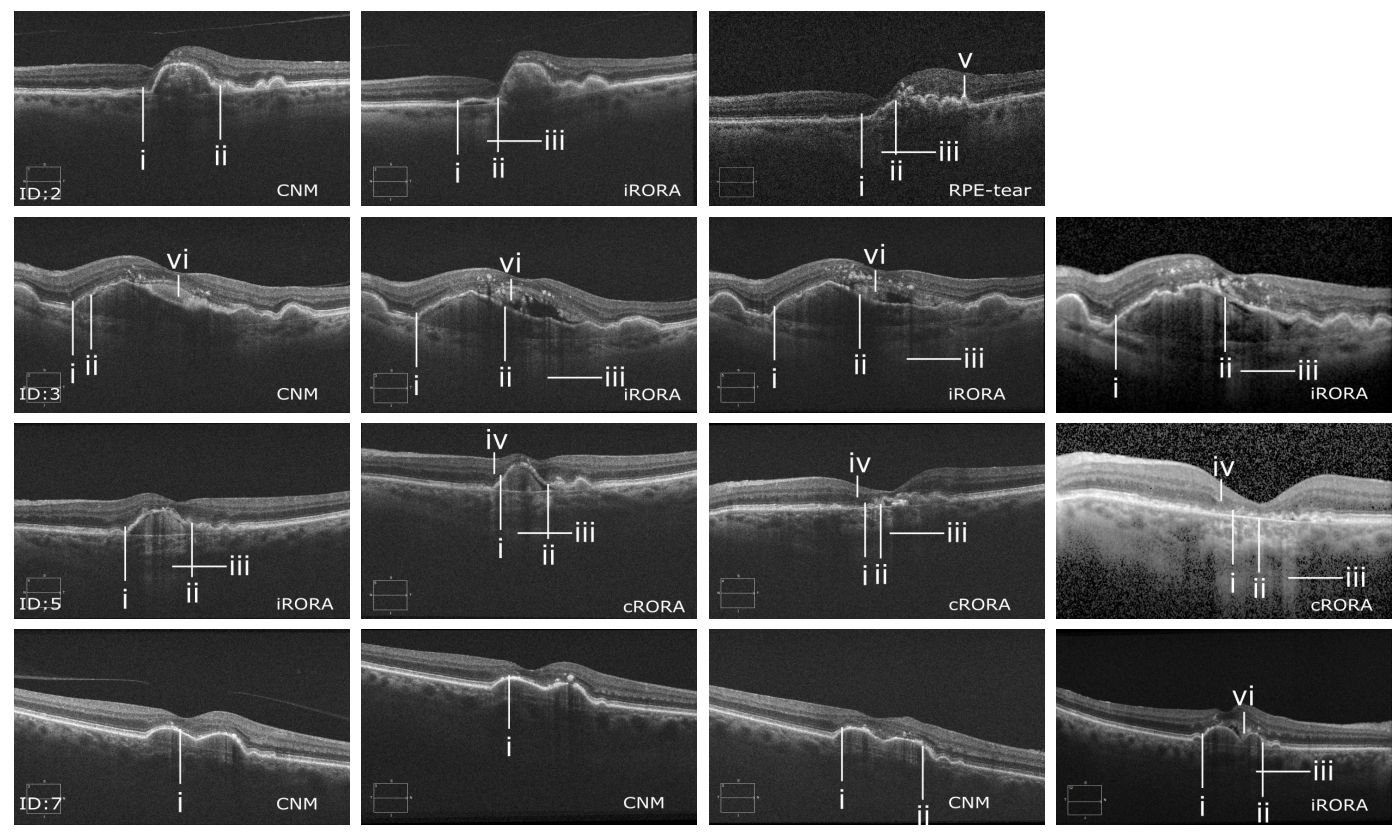

Figure 2 Grading of GA by OCT.

Notes: (i) Photoreceptor degeneration. (ii) Attenuation or disruption of RPE. (iii) Hypertransmission. (iv) Descending outer plexiform layer. (v) Scrolled up RPE. (vi) Subretinal drusenoid material.

Abbreviations: CNM, criteria for iRORA/cRORA not met; cRORA, complete RPE and outer retinal atrophy; ID, patient number; iRORA, incomplete RPE and outer retinal atrophy; RPE, retinal pigment epithelium; OCT, optical coherence tomography; GA, geographic atrophy.

$\mathrm{CI}-1.12,1.56), 0.27 \mathrm{~mm}^{3}(P=0.99,95 \% \mathrm{CI}-0.63,1.17)$, and $0.48 \mathrm{~mm}^{3}(P=0.487,95 \% \mathrm{CI}-0.22,1.18)$ between the treatment and observation groups, respectively. Age of patients was a significant predictor of decreasing PED volume
$(P=0.043,95 \%$ CI $-0.07,-0.001)$. A larger baseline value significantly predicted a larger follow-up PED volume $(P<0.001,95 \%$ CI 1.53, 2.20). Follow-up duration was not a significant predictor $(P=0.433)$. 

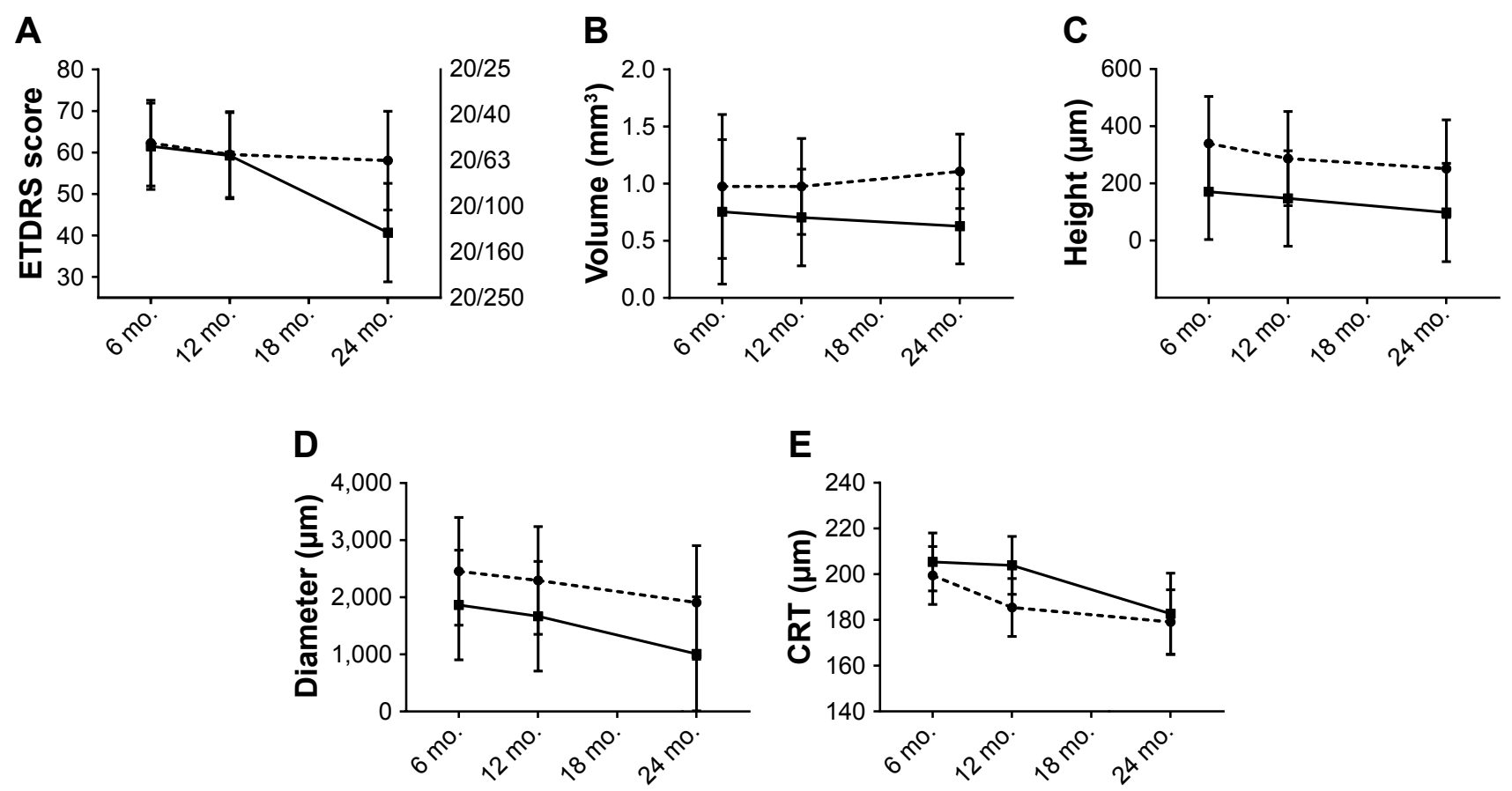

Treatment group

Observation group

Figure 3 Estimated means of BCVA, PED volume, height and diameter, and CRT from 6 to 24 months.

Notes: (A) BCVA; the rightmost $y$-axis corresponds to Snellen equivalent. (B) PED volume. (C) PED height. (D) PED diameter. (E) CRT. The error bars represent $95 \%$ $\mathrm{Cls}$ ( $\mathrm{n}=22$ observations on eight patients).

Abbreviations: BCVA, best-corrected visual acuity; CRT, central retinal thickness; ETDRS, Early Treatment Diabetic Retinopathy Study; mo., months; PED, pigment epithelium detachment.

\section{PED height}

After 24 months of follow-up, estimated means of PED height decreased by 88.3 and $72.8 \mu \mathrm{m}$ in the treatment and observation groups, respectively (Figure 3C). The change of PED height from 6 to 24 months did not differ significantly between the groups $(P=0.842,95 \% \mathrm{CI}-168.6,137.5)$. After 6, 12, and 24 months of follow-up, differences in PED height were $168.8 \mu \mathrm{m}(P=0.949,95 \% \mathrm{CI}-199.7,537.4)$, $139.6 \mu \mathrm{m}(P=0.991,95 \% \mathrm{CI}-229.0,508.1)$, and $153.3 \mu \mathrm{m}$ $(P=0.983,95 \% \mathrm{CI}-226.7,533.2)$ between the treatment and observation groups, respectively. Age of patients, baseline PED height, and duration of follow-up were not significant predictors ( $P=0.483, P=0.075$, and $P=0.188$, respectively).

\section{PED diameter}

After 24 months of follow-up, estimated means of PED diameter decreased by 544 and $856 \mu \mathrm{m}$ in the treatment and observation groups, respectively (Figure 3D). The change of PED diameter from 6 to 24 months did not differ significantly between the groups $(P=0.603,95 \% \mathrm{CI}$ -863, 1,487). After 6,12 , and 24 months of follow-up, differences in PED diameter were $589 \mu \mathrm{m}(P=1.000,95 \%$
CI $-1,478,2,655), 627 \mu \mathrm{m}(P=0.99,95 \% \mathrm{CI}-1,440,2,693)$, and $900 \mu \mathrm{m}(P=0.976,95 \% \mathrm{CI}-1,247,3,048)$ between the treatment and observation groups, respectively. Baseline diameter and duration of follow-up were significant predictors of decreasing PED diameter $(P=0.022$ and $P=0.044$, respectively). Age of patients was not a significant predictor $(P=0.218)$.

\section{Central retinal thickness}

After 24 months of follow-up, estimated means of CRT decreased by 20.3 and $22.7 \mu \mathrm{m}$ in the treatment and observation groups, respectively (Figure 3E). The change in CRT from 6 to 24 months did not differ significantly between the groups $(P=0.852,95 \% \mathrm{CI}-22.8,27.6)$. After 6,12 , and 24 months of follow-up, the differences in CRT were $-5.9 \mu \mathrm{m}$ $(P=1.000,95 \% \mathrm{CI}-35.9,24.0),-18.4 \mu \mathrm{m}(P=0.671,95 \%$ $\mathrm{CI}-48.4,11.5)$, and $-3.5 \mu \mathrm{m}(P=1.000,95 \% \mathrm{CI}-40.8,33.8)$ between the treatment and observation groups, respectively. Age of patients and duration of follow-up were significant predictors of decreasing CRT $(P<0.001$ and $P=0.019$, respectively). A larger baseline value significantly predicted a larger follow-up CRT $(P<0.001)$. 


\section{Geographic atrophy}

GA (defined as iRORA or cRORA) was present in $38 \%$, $75 \%, 71 \%$, and $86 \%$ of patients at baseline and at 6,12 , and 24 months follow-up in both groups combined (Figure 2). After 24 months of follow-up, two patients in the observation group and one patient in the treatment group progressed to cRORA. One patient had identifiable cRORA at baseline in the observation group. A decrease in PED volume was significantly associated with the development of cRORA (Fisher's exact test, $P=0.029$ ).

\section{Safety outcomes}

There were no cases of endophthalmitis or retinal detachment after the intravitreal injections. Two weeks after injection of triamcinolone acetonide, mean IOP was $13 \mathrm{mmHg}$ (ranging from 11 to $16 \mathrm{mmHg}$ ). At baseline, 6 months, 12 months, and 24 months, mean IOP was 16, 15, 13, and $12 \mathrm{mmHg}$ in the treatment group. One patient in the treatment group (Figure 2, ID: 2) was diagnosed with CNV after the first treatment with half-fluence PDT, and was subsequently treated with fullfluence PDT, which led to the development of an RPE tear.

Two patients in the treatment group and one patient in the observation group had an increase of nuclear sclerosis from grade 1 to grade 2 by the end of follow-up. One patient in the observation group (Figure 2, ID: 4) had cataract surgery between 12 and 24 months of follow-up, with no improvement of BCVA because of progression of GA to cRORA.

\section{Discussion}

In the present study, we originally set out to investigate the treatment of both serous and drusenoid aPEDs. However, the referred patients with serous PEDs were either excluded because of $\mathrm{CNV}$ or central serous chorioretinopathy. The higher risk of $\mathrm{CNV}$ in serous PEDs has previously been reported. In a retrospective study, $34 \%$ of serous and $9 \%$ of drusenoid PEDs developed CNV. ${ }^{23}$ In two prospective studies, $32 \%$ of serous aPEDs developed CNV after a mean follow-up of 19.6 months, ${ }^{24}$ and $23 \%$ of drusenoid aPEDs developed CNV over a 5-year period. ${ }^{15}$

Further inclusion and treatment of aPEDs were stopped after the interim analysis of 6 months data. Examination of OCT images showed that $75 \%$ of patients in our study were in different stages of GA formation and progression. ${ }^{25}$ Treatment with anti-VEGF or PDT is not a viable treatment option for GA, and it is still unclear if the use of anti-VEGF agents increases its progression. ${ }^{26}$ PDT has not been shown to directly damage the photoreceptors or RPE. However, PDT induces a selective occlusion of the choriocapillaris, ${ }^{27}$ which in turn could lead to atrophy of RPE and photoreceptors.

BCVA decreased during follow-up with no significant differences between groups. The observation group had a greater, although insignificant, loss of ETDRS letters, probably due to the presence of cRORA in three of four patients. Duration of follow-up was a significant predictor of BCVA loss, and this indicates that this study mainly observed the natural course of drusenoid aPEDs. Our results differ from two prospective studies treating aPEDs with anti-VEGF agents, which resulted in stabilization or improvement of BCVA and no progression of GA or development of CNV. ${ }^{16,17}$ The drusenoid aPEDs in our treatment group were larger (mean $0.98 \mathrm{~mm}^{3}$ ) than in the study conducted by Ritter et al (mean $\left.0.38 \mathrm{~mm}^{3}\right) .{ }^{16}$ Large drusenoid aPEDs are probably a sign of more advanced AMD with worse prognosis. Interestingly, the two largest aPEDs in the present study did not progress to cRORA during follow-up, suggesting that PED volume is not the only risk factor for the progression of GA.

Drusenoid aPEDs are thought to be caused by the confluence of soft drusen over time, ${ }^{5,7}$ which represents a risk factor for progression of GA or development of $\mathrm{CNV}$. In our study, progression of GA to cRORA was identified in $50 \%$ of patients, and development of cRORA was significantly associated with a reduction in PED volume. This is in line with the proposed lifecycle of drusenoid aPEDs with a growth and regression phase that ultimately leads to GA. ${ }^{28,29}$ It has been proposed that an increased distance from the RPE to the choriocapillaris in drusenoid aPEDs has a negative impact on the RPE, leading to RPE atrophy and outer retinal atrophy. ${ }^{30}$ This theory seems plausible as disruptions of the RPE layer and photoreceptors predominantly occur at the apex of drusenoid aPEDs, which is the point of greatest distance to the choriocapillaris. ${ }^{28} \mathrm{~A}$ reduction in PED volume and distance between the choriocapillaris and RPE may improve the metabolism of the RPE and outer retina. However, a reduction in PED volume will probably only have a positive impact on the RPE and the outer retina if it occurs prior to the progression of GA to cRORA. Two studies on rheopheresis (extracorporeal plasma filtration) in the treatment of drusenoid aPEDs have shown interesting results with improvement of BCVA, a decrease of drusenoid PED volume, and a reduced number of patients with progression of GA or development of CNV. ${ }^{31,32}$ 
Future studies evaluating new treatment modalities for drusenoid aPEDs should probably aim to reduce PED volume, and assess with high-resolution OCT if the frequency of GA progression to cRORA is reduced.

\section{Conclusion}

Although limited by the low number of participants, the present study indicates that multitargeted, primarily antiangiogenic therapy does not favorably alter the natural course of large drusenoid aPEDs.

\section{Abbreviations}

Anti-VEGF, anti-vascular endothelial growth factor; aPED, avascular pigment epithelium detachment; BCVA, bestcorrected visual acuity; CNV, choroidal neovascularization; cRORA, complete retinal and outer retina atrophy; CRT, central retinal thickness; ETDRS, Early Treatment Diabetic Retinopathy Study Group; FA, fluorescein angiography; FAF, fundus autofluoresence; GA, geographic atrophy; ICG, indocyanine green angiography; iRORA, incomplete retinal and outer retinal atrophy; nPED, neovascular pigment epithelium detachment; OCT, optical coherence tomography; PDT, photodynamic therapy.

\section{Data sharing statement}

The datasets used and analyzed during the current study are available from the corresponding author on request.

\section{Acknowledgment}

This project has been made possible by the Norwegian ExtraFoundation for Health and Rehabilitation (2016/FO80635). The sponsor had no involvement in any of the stages from study design to submission.

\section{Disclosure}

The authors report no conflicts of interest in this work.

\section{References}

1. Klein BE, Klein R. Cataracts and macular degeneration in older Americans. Arch Ophthalmol. 1982;100(4):571-573.

2. Solomon SD, Lindsley K, Vedula SS, Krzystolik MG, Hawkins BS. Antivascular endothelial growth factor for neovascular age-related macular degeneration. Cochrane Database Syst Rev. 2014;8:CD005139.

3. Bourne RR, Jonas JB, Flaxman SR, et al. Prevalence and causes of vision loss in high-income countries and in eastern and central Europe: 1990-2010. Br J Ophthalmol. 2014;98(5):629-638.

4. Schmidt-Erfurth U, Waldstein SM. A paradigm shift in imaging biomarkers in neovascular age-related macular degeneration. Prog Retin Eye Res. 2016;50:1-24.

5. Zayit-Soudry S, Moroz I, Loewenstein A. Retinal pigment epithelial detachment. Surv Ophthalmol. 2007;52(3):227-243.
6. Bird AC. Doyne lecture. Pathogenesis of retinal pigment epithelial detachment in the elderly; the relevance of Bruch's membrane change. Eye (Lond). 1991;5(Pt 1):1-12.

7. Casswell AG, Kohen D, Bird AC. Retinal pigment epithelial detachments in the elderly: classification and outcome. Br J Ophthalmol. 1985; 69(6):397-403.

8. Roquet W, Roudot-Thoraval F, Coscas G, Soubrane G. Clinical features of drusenoid pigment epithelial detachment in age related macular degeneration. Br J Ophthalmol. 2004;88(5):638-642.

9. Lee DK, Kim SH, You YS, Kwon OW. High dose intravitreal bevacizumab for refractory pigment epithelial detachment in age-related macular degeneration. Korean J Ophthalmol. 2016;30(4):265-271.

10. Dirani A, Ambresin A, Marchionno L, Decugis D, Mantel I. Factors influencing the treatment response of pigment epithelium detachment in age-related macular degeneration. Am J Ophthalmol. 2015;160(4): 732-738.

11. Arora S, McKibbin M. One-year outcome after intravitreal ranibizumab for large, serous pigment epithelial detachment secondary to age-related macular degeneration. Eye (Lond). 2011;25(8):1034-1038.

12. Baba T, Kitahashi M, Kubota-Taniai M, Oshitari T, Yamamoto S. Two-year course of subfoveal pigment epithelial detachment in eyes with age-related macular degeneration and visual acuity better than 20/40. Ophthalmologica. 2012;228(2):102-109.

13. Verteporfin in Photodynamic Therapy Study Group. Verteporfin therapy of subfoveal choroidal neovascularization in age-related macular degeneration: two-year results of a randomized clinical trial including lesions with occult with no classic choroidal neovascularization - verteporfin in photodynamic therapy report 2. Am J Ophthalmol. 2001;131(5):541-560.

14. Axer-Siegel R, Ehrlich R, Rosenblatt I, et al. Photodynamic therapy for occult choroidal neovascularization with pigment epithelium detachment in age-related macular degeneration. Arch Ophthalmol. 2004; 122(4):453-459.

15. Cukras C, Agrón E, Klein ML, et al. Natural history of drusenoid pigment epithelial detachment in age-related macular degeneration: agerelated eye Disease Study report no 28. Ophthalmology. 2010;117(3): 489-499.

16. Ritter M, Bolz M, Sacu S, et al. Effect of intravitreal ranibizumab in avascular pigment epithelial detachment. Eye (Lond). 2010;24(6):962-968.

17. Gallego-Pinazo R, Marina A, Suelves-Cogollos AM, et al. Intravitreal ranibizumab for symptomatic drusenoid pigment epithelial detachment without choroidal neovascularization in age-related macular degeneration. Clin Ophthalmol. 2011;5:161-165.

18. Krishnan R, Lochhead J. Regression of soft drusen and drusenoid pigment epithelial detachment following intravitreal anti-vascular endothelial growth factor therapy. Can J Ophthalmol. 2010;45(1):83-84.

19. Kim S, Oh J, Kim K. Morphologic changes in patient with drusen and drusenoid pigment epithelial detachment after intravitreal ranibizumab for choroidal neovascular membrane: a case report. Open Ophthalmol J. 2016;10:1-4.

20. Novais EA, Badaró E, Regatieri CV, Duker J, de Oliveira Bonomo PP. Regression of drusen after combined treatment using photodynamic therapy with verteporfin and ranibizumab. Ophthalmic Surg Lasers Imaging Retina. 2015;46(2):275-278.

21. Lee NY, Kim KS. Photodynamic therapy treatment for eyes with drusenoid pigment epithelium detachment. Korean J Ophthalmol. 2008; 22(3):194-196.

22. Sadda SR, Guymer R, Holz FG, et al. Consensus definition for atrophy associated with age-related macular degeneration on OCT: classification of atrophy report 3. Ophthalmology. 2018;125(4):537-548.

23. Hartnett ME, Weiter JJ, Garsd A, Jalkh AE. Classification of retinal pigment epithelial detachments associated with drusen. Graefes Arch Clin Exp Ophthalmol. 1992;230(1):11-19.

24. Elman MJ, Fine SL, Murphy RP, Patz A, Auer C. The natural history of serous retinal pigment epithelium detachment in patients with agerelated macular degeneration. Ophthalmology. 1986;93(2):224-230. 
25. Wu Z, Luu CD, Ayton LN, et al. Optical coherence tomography-defined changes preceding the development of drusen-associated atrophy in age-related macular degeneration. Ophthalmology. 2014;121(12): 2415-2422.

26. Abdelfattah NS, Zhang H, Boyer DS, Sadda SR. Progression of macular atrophy in patients with neovascular age-related macular degeneration undergoing antivascular endothelial growth factor therapy. Retina. 2016;36(10):1843-1850.

27. Schlötzer-Schrehardt U, Viestenz A, Naumann GO, Laqua H, Michels S, Schmidt-Erfurth U. Dose-related structural effects of photodynamic therapy on choroidal and retinal structures of human eyes. Graefes Arch Clin Exp Ophthalmol. 2002;240(9):748-757.

28. Balaratnasingam C, Yannuzzi LA, Curcio CA, et al. Associations between retinal pigment epithelium and drusen volume changes during the lifecycle of large drusenoid pigment epithelial detachments. Invest Ophthalmol Vis Sci. 2016;57(13):5479-5489.
29. Schlanitz FG, Baumann B, Kundi M, et al. Drusen volume development over time and its relevance to the course of age-related macular degeneration. Br J Ophthalmol. 2017;101(2):198-203.

30. Mrejen S, Sarraf D, Mukkamala SK, Freund KB. Multimodal imaging of pigment epithelial detachment: a guide to evaluation. Retina. 2013; 33(9):1735-1762.

31. Blaha M, Rencova E, Langrova H, et al. Rheohaemapheresis in the treatment of nonvascular age-related macular degeneration. Atheroscler Suppl. 2013;14(1):179-184.

32. Koss MJ, Kurz P, Tsobanelis T, et al. Prospective, randomized, controlled clinical study evaluating the efficacy of Rheopheresis for dry age-related macular degeneration. Dry AMD treatment with Rheopheresis Trial-ART. Graefes Arch Clin Exp Ophthalmol. 2009;247(10): 1297-1306.
Clinical Ophthalmology

\section{Publish your work in this journal}

Clinical Ophthalmology is an international, peer-reviewed journal covering all subspecialties within ophthalmology. Key topics include: Optometry; Visual science; Pharmacology and drug therapy in eye diseases; Basic Sciences; Primary and Secondary eye care; Patient Safety and Quality of Care Improvements. This journal is indexed on

Submit your manuscript here: http://www.dovepress.com/clinical-ophthalmology-journal

\section{Dovepress}

PubMed Central and CAS, and is the official journal of The Society of Clinical Ophthalmology (SCO). The manuscript management system is completely online and includes a very quick and fair peer-review system, which is all easy to use. Visit http://www.dovepress.com/ testimonials.php to read real quotes from published authors. 